\title{
TWO HELLY TYPE THEOREMS
}

\author{
KRZYSZTOF KOLODZIEJCZYK
}

\begin{abstract}
Two Helly type theorems for convex sets containing $k$-dimensional half-flats are established, one of them being an extension of Katchalski's theorem [3] where the case $k=1$ was considered.
\end{abstract}

1. Introduction. For any set $C$ in linear space let conv $C$ denote the convex hull of $C$, aff $C$ the affine hull of $C$, and $\operatorname{dim} C$ the dimension of aff $C$. By a $k$-flat ( $k$-half-flat) we mean a translate of a subspace (half-space) of dimension $k$.

In this note we prove the following two Helly type theorems.

THEOREM 1. If $\mathscr{F}$ is a finite family of convex sets in $\mathbf{R}^{n}$ such that the intersection of any $2 n-2 k+2$ members of $\mathscr{F}$ contains a $k$-half-flat, then $\cap \mathcal{F}$ contains a $k$-half-flat.

THEOREM 2. If $\mathscr{F}$ is a finite family of convex sets in a linear space such that:

(1) $\max \{\operatorname{dim} C \mid C \in \mathscr{F}\}=d$,

(2) $\operatorname{dim} \cup \mathcal{F}=n$,

(3) card $\mathscr{F} \geqslant r(n, d, k)$,

where

$$
r(n, d, k)= \begin{cases}2 d-2 k+2 & \text { if } 1 \leqslant k \leqslant d, n=d, \\ 2 d-2 k+1 & \text { if } 1 \leqslant k<d, n>d, \\ 2 & \text { if } k=d, n>d,\end{cases}
$$

then $\cap \mathcal{F}$ contains a $k$-half-flat provided the intersection of any $r(n, d, k)$ members of $\mathscr{F}$ contains a $k$-half-flat.

The case $k=1$ in Theorem 1 was proved by Katchalski [3]. Our proof of Theorem 1 employs Katchalski's result and the following theorem of de Santis [2].

If $\mathcal{F}$ is a finite family of convex sets in $\mathbf{R}^{n}$ such that the intersection of any $n-k+1$ members of $\mathcal{F}$ contains a $k$-flat, then $\cap \mathcal{F}$ contains a $k$-flat.

Theorem 2 is analogous to the results by Netrebin [4, Theorems 1 and 2].

Related Helly type theorems can be found in [1].

2. Proof of Theorem 1. The validity of the theorem for $k=1$ follows by Katchalski's result.

Let $1<k \leqslant n$. Since $2 n-2 k+2 \geqslant n-(k-1)+1$, by our assumption and the theorem of de Santis there exists a $(k-1)$-flat $\mathbf{H}$ such that

$$
\mathbf{H} \subset \bigcap \mathcal{F} .
$$

Received by the editors April 11, 1983 and, in revised form, June 21, 1983.

1980 Mathematics Subject Classification. Primary 52A35, 52A20.

$\mathrm{Key}$ words and phrases. Convex set, $k$-dimensional half-flat, Helly's theorem 
Now let $\mathbf{H}^{*}$ be an $(n-k+1)$-flat which is complementary to $\mathbf{H}$ and let

$$
\mathscr{F}^{*}=\left\{C^{*}=C \cap \mathbf{H}^{*} \mid C \in \mathscr{F}\right\}
$$

be a family of convex sets in $\mathbf{H}^{*}$. Choose any subfamily $\mathscr{F}_{1}, \mathscr{F}_{1} \subset \mathscr{F}_{\text {, }}$, consisting of $2 n-2 k+2$ elements. By assumption there exists a $k$-half-flat $\mathbf{E}$ such that $\mathbf{E} \subset \cap \mathscr{F}_{1}$. It is clear that $\mathbf{E} \cap \mathbf{H}^{*}$ contains a 1-half-flat (ray). This shows that any subfamily $\mathscr{F}_{1}^{*}, \mathscr{F}_{1}^{*} \subset \mathscr{F}^{*}$, consisting of $2 n-2 k+2$ elements, contains a ray in its intersection, and we can apply Katchalski's theorem to the family $\mathscr{F}^{*}$ in $\mathbf{H}^{*}$. This way we infer that there exists a ray $\lambda$ which is contained in all members of $\mathscr{F}^{*}$ and consequently in all members of $\mathcal{F}$. Without loss of generality we can suppose that an apex of $\lambda$ belongs to $\mathbf{H}$. Now obviously the set $\operatorname{conv}(\mathbf{H} \cup \lambda)$ is a $k$-half-flat and is contained in $\cap \mathscr{F}$. This completes the proof.

3. Proof of Theorem 2. In the case of $1 \leqslant k \leqslant d, n=d$, Theorem 2 coincides with Theorem 1. The other two cases we prove by induction on card $\mathscr{F}$. Let $s=\operatorname{card} \mathscr{F}$ and $r=r(n, d, k)$. The theorem is obviously true for $s=r$. Suppose the result holds for $s=s_{0} \geqslant r$ and take $s=s_{0}+1$. Putting

$$
B_{m}=\bigcap\left\{C_{i} \mid i \neq m\right\}, \quad m=1,2, \ldots, s_{0}+1,
$$

it can be shown, similarly as in the proof of Theorem 2 in [4], that in both cases considered

$$
\operatorname{dim} \bigcup\left\{B_{m} \mid m=1,2, \ldots, s_{0}+1\right\}=d .
$$

Now let

$$
\mathbf{B}=\operatorname{aff}\left(\bigcup\left\{B_{m} \mid m=1,2, \ldots, s_{0}+1\right\}\right) .
$$

Case $1.1 \leqslant k<d, n>d$. Since $n>d$ there is a set, say $C_{1}$, contained in $\mathscr{F}$ such that $\operatorname{dim}\left(C_{1} \cap \mathbf{B}\right) \leqslant d-1$. Consider the family $\mathscr{F}^{\prime}=\left\{C^{\prime}=\mathbf{P} \cap C \mid C \in \mathscr{F}\right\}$ of convex sets in $\mathbf{P}=\operatorname{aff}\left(C_{1} \cap \mathbf{B}\right)$. Choose any subfamily $\mathscr{F}_{1}^{\prime}, \mathscr{F}_{1}^{\prime} \subset \mathscr{F}^{\prime}$, containing $2 d-2 k$ sets. The following inclusions are obvious:

$$
\bigcap \mathscr{F}_{1}^{\prime}=\bigcap \mathscr{F}_{1} \cap \mathbf{P} \supset B_{m_{0}} \cap \mathbf{P} \supset B_{m_{0}}
$$

for some $m_{0}, 2 \leqslant m_{0} \leqslant s_{0}+1$. Our induction assumption implies that the set $B_{m_{0}}$ contains a $k$-half-flat. Hence, by the above inclusions, any subfamily $\mathscr{F}_{1}^{\prime}$ consisting of $2 d-2 k$ sets contains a $k$-half-flat in its intersection. This shows that the family $\mathcal{F}^{\prime}$ satisfies the conditions of Theorem 1 in the $(d-1)$-flat $\mathbf{P}$ and hence there is a $k$-half-flat which is contained in $\cap \mathcal{F}^{\prime}$ and consequently in $\cap \mathscr{F}$.

Case 2. $k=d, n>d$. Consider the family $\mathscr{F}^{\prime \prime}=\left\{C^{\prime \prime}=\mathbf{B} \cap C \mid C \in \mathscr{F}\right\}$. Choose any two sets of family $\mathcal{F}^{\prime \prime}$ (e.g. $C_{1}^{\prime \prime}$ and $\left.C_{2}^{\prime \prime}\right)$. Then we have

$$
C_{1}^{\prime \prime} \cap C_{2}^{\prime \prime}=\mathbf{B} \cap\left(C_{1} \cap C_{2}\right) \supset \mathbf{B} \cap B_{m_{0}} \supset B_{m_{0}}
$$

for some $m_{0}, 3 \leqslant m_{0} \leqslant s_{0}+1$. This shows, by our induction hypothesis, that the family $\mathcal{F}^{\prime \prime}$ satisfies the conditions of Theorem 1 in the $d$-flat B. Now applying Theorem 1 we complete the proof of Theorem 2.

REMARK. A simple modification of the example given in the last part of the proof of Theorem 2 in [4] shows that the number $r(n, d, k)$ cannot be replaced by a smaller number. 


\section{REFERENCES}

1. L. Danzer, B. Grünbaum and V. Klee, Helly's theorem and its relatives, Convexity, Proc. Sympos. Pure Math., Vol. 7, Amer. Math. Soc., Providence, R. I., pp. 101-180.

2. R. de Santis, $A$ generalization of Helly's theorem, Proc. Amer. Math. Soc. 8 (1957), 336-340.

3. M. Katchalski, A Helly type theorem for convex sets, Canad. Math. Bull. 21 (1978), 121-123.

4. A. G. Netrebin, Properties of systems of n-dimensional convex sets in finite-dimensional linear spaces, Mat. Z. 25 (1979), 603-618; English transl., Math. Notes 25 (1979), 312-320.

Institute of Mathematics, Technical University of Wroclaw, Wybrzeze Wyspiańskiego 27, 50-370 Wroclaw, Poland 